hep-th/9610154

UTTG-15-96

TUW-96/21

JHU-TIPAC-96020

\title{
Searching for K3 Fibrations
}

\author{
A.C. Avram $^{1 \mathrm{a}}$, M. Kreuzer ${ }^{2 \mathrm{~b}}$, M. Mandelberg ${ }^{3 \mathrm{c}}$ and H. Skarke ${ }^{2 \mathrm{~d}}$ \\ ${ }^{1}$ Theory Group, Physics Department, University of Texas at Austin \\ Austin, TX 78712, USA \\ ${ }^{2}$ Institut für Theoretische Physik, Technische Universität Wien \\ Wiedner Hauptstraße 8-10, A-1040 Wien, AUSTRIA \\ ${ }^{3}$ The Johns Hopkins University, Baltimore, MD, 21218, USA
}

\begin{abstract}
We present two methods for studying fibrations of Calabi-Yau manifolds embedded in toric varieties described by single weight systems. We analyse 184,026 such spaces and identify among them 124,701 which are K3 fibrations. As some of the weights give rise to two or three distinct types of fibrations, the total number we find is 167,406 . With our methods one can also study elliptic fibrations of 3-folds and K3 surfaces. We also calculate the Hodge numbers of the 3 -folds obtaining more than three times as many as were previously known.
\end{abstract}

October 1996

\footnotetext{
${ }^{\mathrm{a}}$ e-mail: alex@physics.utexas.edu

be-mail: kreuzer@tph16.tuwien.ac.at

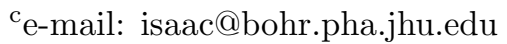

de-mail:skarke@tph16.tuwien.ac.at
} 


\section{Introduction}

Ever since Calabi-Yau manifolds entered into the lexicon of string theory, there have been various ways to classify them. Initially, it was (and, of course, still is) of great interest to calculate their Hodge numbers, thereby predicting the number of massless families of matter fields. This was of particular interest, since in the early days of the study of Calabi-Yau manifolds, there were few known examples, and so it seemed that it might be difficult to find one with Euler number $\left|\chi_{E}\right|=6$. By now there are dozens of topologically distinct Calabi-Yau manifolds with $\left|\chi_{E}\right|=6$, and in any case, these days we are happy to have manifolds with other values of $\chi_{E}$. They provide string theorists with a wide assortment of laboratories in which to test their ideas. In the beginning, there were $\mathbb{P}(4 \mid 5), \mathbb{P}(5 \mid 42), \mathbb{P}(5 \mid 33), \mathbb{P}(6 \mid 322)$, $\mathbb{P}(7 \mid 2 \quad 222)$ and the $\mathbb{Z}_{3}$ orbifold [1]. Soon after followed complete intersections of polynomial hypersurfaces in direct products of projective spaces [2,3], and then transverse polynomials in weighted projective spaces $[4,5]$. Most recently Calabi-Yau manifolds have been constructed as embeddings in toric varieties [6]. Using this approach, it was shown in $[7,8]$ that there are 184,026 distinct sets of weights that define spaces in which Calabi-Yau manifolds can be embedded. These include both the weights that admit transverse polynomials, as well as those that don't, but nonetheless lead to reflexive polyhedra and thereby to varieties allowing smooth Calabi-Yau hypersurfaces. The purpose of this paper is to provide further means of analysing these manifolds.

Among the dualities that have been recently studied is that between the $E_{8} \times E_{8}$ heterotic string compactified on $K 3 \times T^{2}$ and the Type IIA string compactified on a Calabi-Yau manifold. In [9] specific examples for this Calabi-Yau manifold were proposed. They rely on two pieces of evidence in support of the conjectured duality. The first is that the massless spectra on both sides match at generic points of their moduli spaces. The second involves a close examination of certain regions of the moduli space of the Calabi-Yau manifold. This analysis suggests a natural identification of one of the Kähler moduli with the heterotic dilaton. Specifically, the authors analyze the heterotic string with the $T^{2}$ chosen so that classically there is a single extra $S U(2)$ at the self-dual point, $\tau=i$. This suggests that when quantum effects are included, this singularity will split into two points where hypermulitplets become massless, much as in $N=2$ QFT [10].

As it turns out, there is a Calabi-Yau manifold that has the correct Hodge numbers. The only known Calabi-Yau manifold with $h_{11}=2$ and $h_{21}=128$ is $\mathrm{M}=\mathbb{P}_{4}^{1,1,2,2,6}[12]$. It is impressive that $\mathrm{M}$ also has a moduli space that resembles the Seiberg-Witten solution. One of the keys to this correspondence comes from mirror symmetry. By analysing the mirror map for $M \leftrightarrow \mathrm{W}^{1}$, it can be shown that $\mathrm{M}$ has a Kähler modulus that "happens" to have the modular transformation properties appropriate for a parameter describing the moduli space of an $N=2$ theory with $S U(2)$ gauge symmetry. It was suggested in [11] that this is a generic feature of Calabi-Yau manifolds that are $K 3$ fibrations. Indeed it is not difficult to see that $\mathbb{P}_{4}^{1,1,2,2,6}[12]$ is a $K 3$ fibration with standard fiber given by $\mathbb{P}_{3}^{1,1,1,3}$. A short list of fibrations was provided in [11].

\footnotetext{
1 W can be chosen as $\mathbb{P}_{4}^{12,13,18,25,28}[96]$.
} 
Of these, 31 are transverse hypersurfaces in $\mathbb{P}_{4}^{\mathbf{k}} \cdot{ }^{2}$ The authors proceed as follows. Consider a $K 3$ given by a hypersurface in $\mathbb{P}_{3}^{\mathbf{k}}$. If $\mathbf{k}=\left(1, k_{2}, k_{3}, k_{4}\right)$, then it is easy to see how to form a $K 3$ fibration in $\mathbb{P}_{4}^{1,1,2 k_{2}, 2 k_{3}, 2 k_{4}}$. The $\mathbb{P}_{1}$ base space is given by the ratio of the two coordinates with weight one, while the $K 3$ is as above. Of the $95 \mathrm{~K} 3$ 's that are given as a hypersurface in a $\mathbb{P}_{3}^{\mathbf{k}}, 41$ have a coordinate with weight one. Of these, 31 can generate a transverse polynomial in $\mathbb{P}_{4}^{\mathbf{k}} \cdot{ }^{3}$ These results were extended in [12]. That article describes the general problem of determining whether a given $\mathbb{P}_{4}^{\mathbf{k}}$ is a $K 3$ fibration. The authors were able to identify $628 K 3$ fibrations among the $7,555 \mathbb{P}_{4}^{\mathbf{k}}$ that admit transverse polynomials.

The question of why $K 3$ fibrations are so important to heterotic-Type II duality has been largely laid to rest by Aspinwall and Louis in [13]. Their result is that, assuming the Type IIA dual of the weakly coupled heterotic string is in the Calabi-Yau phase, then this Calabi-Yau manifold must be a $K 3$ fibration. They achieve this result as a consequence of demanding that the pre-potentials for both sides match.

Given this result, it has become useful to identify those Calabi-Yau manifolds that are $K 3$ fibrations. We do this using the methods of toric geometry. In Section 2 we briefly review some elements of toric geometry. In Section 3 we describe in detail how a $K 3$ fibration is manifested in a polyhedron. In [14] several such examples were analyzed. It was noted that in each case, the Newton polyhedron for the Calabi-Yau manifold exhibited the fibration in a particularly simple manner, to wit, there was a hyperplane through the origin whose intersection with the polyhedron gave a reflexive three dimensional subpolyhedron ${ }^{4}$. We will show that the general case is more complicated. Any projection of the 4 dimensional polyhedron onto a 3 dimensional sublattice that produces a reflexive polyhedron defines a $K 3$ fibration. This is explained and demonstrated by examples. We also outline our approach for identifying fibrations. Section 4 provides the technical details. We explain how a certain kind of polyhedron known as a maximal Newton polyhedron has the property that if it has a face that is a reflexive 3 dimensional polyhedron, then there is always a unique projection onto that face. We also explain our second strategy, which is somewhat complementary to the first, and also relies on some simple geometric properties of how faces behave under projection. We summarize our results in Section 5. Of the 184,026 polyhedra that we analyze, we identify 124,701 that are $K 3$ fibrations, 5,130 that are not, and 54,195 that do not yield to our methods. As an indication of the efficiency of our approaches, we note that among the 7,555 transverse weights we find 5,370 models with one, two or three fibrations (to be compared with the 628 fibrations of [12]). Finally, we have calculated the Hodge numbers for all 184,026 polyhedra. We find 14,121 different pairs of Hodge numbers, thereby increasing the number of known pairs by a factor of more than three.

\footnotetext{
2 Another 25 are intersections of two hypersurfaces in $\mathbb{P}_{5}^{\mathbf{k}}$.

3 If we allow for the $\mathbb{P}_{4}^{\mathbf{k}}$ 's described in $[7,8]$, then this approach "saturates the bound", and all 41 of these K3's produce fibrations.

4 It was first conjectured in [11] that the polyhedron of the K3 should be a subpolyhedron of the polytope associated with the Calabi-Yau family.
} 


\section{Calabi-Yau hypersurfaces in toric varieties}

In toric geometry algebraic varieties are described with the help of a dual pair of lattices $N$ and $M$, each isomorphic to $\mathbb{Z}^{n}$, and a fan $\Sigma$ defined on $N_{\mathbb{R}}$ (the real extension of the $N$ lattice). Then there are various ways of constructing the variety $V_{\Sigma}$ from the toric data.

In the old "gluing" approach, affine varieties $V_{\sigma}$ are associated with each cone $\sigma \in \Sigma$, and $V_{\Sigma}$ is obtained by gluing them together in a certain way (see, for example, [15]).

In the holomorphic quotient approach [16] it is possible to assign a single homogeneous coordinate system to $V_{\Sigma}$ in a way similar to the usual construction of $\mathbb{P}^{n}$. To this end one assigns a coordinate $z_{k}, k=1, \cdots, N$ (hopefully no confusion will arise from the two different usages of the symbol $N$ ) to each one dimensional cone in $\Sigma$. If the primitive generators $v_{1}, \cdots, v_{N}$ of these one dimensional cones span $N_{\mathbb{R}}$ (the real extension of $N$ ), then there must be $N-n$ independent linear relations of the type $\sum_{k} w_{j}^{k} v_{k}=0$. These linear relations are used to define equivalence relations of the type

$$
\left(z_{1}, \cdots, z_{N}\right) \sim\left(\lambda^{w_{j}^{1}} z_{1}, \cdots, \lambda^{w_{j}^{N}} z_{N}\right), \quad j=1, \cdots, N-n
$$

on the space $\mathbb{C}^{N}-Z_{\Sigma}$. The set $Z_{\Sigma}$ is determined by the fan $\Sigma$ in the following way: It is the union of spaces $\left\{\left(z_{1}, \cdots, z_{N}\right): z_{i}=0 \forall i \in I\right\}$, where the index sets $I$ are those sets for which $\left\{v_{i}: i \in I\right\}$ does not belong to a cone in $\Sigma$. Thus $\left(\mathbb{C}^{*}\right)^{N} \subset \mathbb{C}^{N} \backslash Z_{\Sigma} \subset \mathbb{C}^{N} \backslash\{0\}$. Then $V_{\Sigma}=\left(\mathbb{C}^{N} \backslash Z_{\Sigma}\right) /\left(\mathbb{C}^{*}\right)^{(N-n)}$, where the $N-n$ groups $\mathbb{C}^{*}$ act by the equivalence relations given above.

A third approach is the symplectic quotient construction [17], which is closely related to the holomorphic quotient construction. Here each of the $\mathbb{C}^{*}$ s s is decomposed as $\mathbb{R}_{+} \times U(1)$. One first chooses representatives of the $\mathbb{R}_{+}^{N-n}$ equivalence classes by imposing $N-n$ equations $\sum_{k} w_{j}^{k}\left|z_{k}\right|^{2}=r_{j}$, and then the resulting space is divided by the remaining $(U(1))^{N-n}$ invariance.

There are several reasons why we are mainly interested in the holomorphic/symplectic quotient approach:

- The holomorphic quotient construction leads immediately to the usual descriptions of projective spaces and is closely related to weighted projective spaces.

- Witten's gauged linear sigma model [18] leads automatically to the symplectic quotient construction.

- Describing Calabi-Yau spaces with Batyrev's method [6], the generators of one dimensional cones are just the integer points of the dual polyhedron $\Delta^{*}$ in the $N$ lattice.

- The description of K3 fibrations of Calabi-Yau spaces is far easier with these constructions.

Let us briefly outline the construction of a Calabi-Yau hypersurface in a space described by a reflexive polyhedron: We take $\Delta$ to be a reflexive polyhedron in $M_{\mathbb{R}}$ (for example the 
Newton polyhedron of some weighted projective space), $\Delta^{*} \subset N_{\mathbb{R}}$ its dual, and $\Sigma$ a fan defined by a maximal triangulation of $\Delta^{*}$. This means that the integer generators $v_{1}, \cdots, v_{N}$ of the one dimensional cones are just the integer points (except the origin) of $\Delta^{*}$. The polynomial that determines the CY-hypersurface takes the form

$$
\sum_{x \in \Delta \cap M} a_{x} \prod_{k=1}^{N} z_{k}^{\left\langle v_{k}, x\right\rangle+1} .
$$

It is easily checked that it is quasihomogeneous with respect to all $N-n$ relations of (1) with degrees $d_{j}=\sum_{k=1}^{N} w_{j}^{k}, j=1, \cdots N-n$. Note how the reflexivity of the polyhedron ensures that the exponents are nonnegative.

The Hodge numbers $h_{11}$ and $h_{21}$ for 3 dimensional CY-hypersurfaces of this type are [6]

$$
h_{11}=l\left(\Delta^{*}\right)-5-\sum_{\operatorname{codim} \theta^{*}=1} l^{*}\left(\theta^{*}\right)+\sum_{\operatorname{codim} \theta^{*}=2} l^{*}\left(\theta^{*}\right) l^{*}(\theta)
$$

and

$$
h_{21}=l(\Delta)-5-\sum_{\operatorname{codim} \theta=1} l^{*}(\theta)+\sum_{\operatorname{codim} \theta=2} l^{*}\left(\theta^{*}\right) l^{*}(\theta),
$$

where $\theta$ and $\theta^{*}$ are faces of $\Delta$ and $\Delta^{*}$, respectively, and $l(\cdot)$ and $l^{*}(\cdot)$ denote the numbers of integer points and integer interior points of polytopes. These formulas are invariant under the simultaneous exchange of $\Delta$ with $\Delta^{*}$ and $h_{11}$ with $h_{21}$ so that Batyrev's construction is manifestly mirror symmetric (at least at the level of spectra).

\section{Toric K3 fibrations}

In the previous section we have seen how the polyhedron $\Delta^{*}$ determines the fan in the $N$ lattice which is used to describe the ambient space, whereas the polyhedron $\Delta$ describes the polynomial whose vanishing locus is our CY hypersurface. For the description of a fibration, we need the interplay between both sides. The following theorem relates properties of $\Delta$ and $\Delta^{*}$ which, as we will soon see, are essential for the construction of a fibration whose base space is $\mathbb{P}^{1}$ and whose generic fiber is an $n-1$ dimensional $\mathrm{CY}$ space.

Theorem: Let $\Delta$ be an $\mathrm{n}$ dimensional reflexive polyhedron in $M_{\mathbb{R}}$. Then the following statements are equivalent:

(1) There exists a projection operator $P: \quad M \rightarrow M_{n-1}$, where $M_{n-1}$ is an $n-1$ dimensional sublattice, such that $P \Delta$ is a reflexive polyhedron in $M_{n-1}$.

(2) There is a lattice hyperplane in $N_{\mathbb{R}}$ through the origin whose intersection with $\Delta^{*}$ is an $n-1$ dimensional reflexive polyhedron.

The respective $n-1$ dimensional polyhedra are dual to one another.

Proof: For showing that (1) implies (2), we choose a lattice basis $e_{1}, \cdots, e_{n}$ for $M$ such that $P e_{i}=e_{i}$ for $i<n, P e_{n}=0$ (such a basis exists because $P$ projects onto a sublattice). We denote coordinates with respect to this basis by $\left(x^{i}\right)$ and coordinates with respect to the dual 

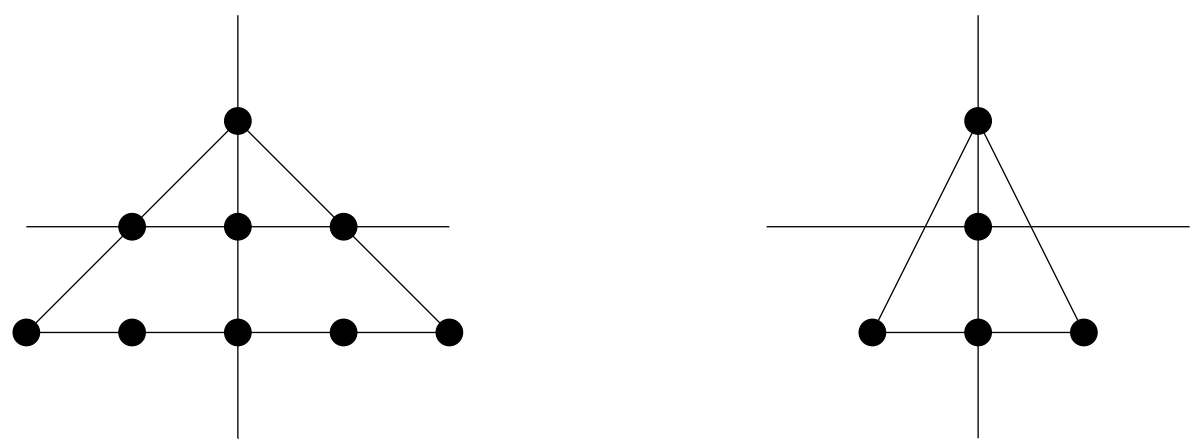

Figure 1: A dual pair of reflexive polygons

basis $e^{j}$ of $N$ by $\left(y_{j}\right)$. For $(2) \Rightarrow(1)$ we start with a basis $e^{j}$ of $N$ such that $\Delta^{*} \cap\left\{y_{n}=0\right\}$ is reflexive and define $e_{i}$ to be the dual basis. In both cases we define

$$
\left.\Delta_{n-1}=\left\{\left(x^{1}, \cdots, x^{n-1}\right): \exists x^{n} \text { with }\left(x^{1}, \cdots, x^{n-1}, x^{n}\right) \in \Delta\right)\right\}
$$

and

$$
\left(\Delta^{*}\right)_{n-1}=\left\{\left(y_{1}, \cdots, y_{n-1}\right):\left(y_{1}, \cdots, y_{n-1}, 0\right) \in \Delta^{*}\right\} .
$$

Now we note that we can prove both directions if we manage to show that $\Delta_{n-1}$ and $\left(\Delta^{*}\right)_{n-1}$ are dual. This is the case, due to

$$
\begin{aligned}
\left(\Delta_{n-1}\right)^{*} & =\left\{\left(y_{1}, \cdots, y_{n-1}\right):\left\langle\left(y_{1}, \cdots, y_{n-1}\right),\left(x^{1}, \cdots, x^{n-1}\right)\right\rangle \geq-1 \forall\left(x^{1}, \cdots, x^{n-1}\right) \in \Delta_{n-1}\right\} \\
& =\left\{\left(y_{1}, \cdots, y_{n-1}\right):\left\langle\left(y_{1}, \cdots, y_{n-1}, 0\right),\left(x^{1}, \cdots, x^{n}\right)\right\rangle \geq-1 \forall\left(x^{1}, \cdots, x^{n}\right) \in \Delta\right\} \\
& =\left(\Delta^{*}\right)_{n-1} .
\end{aligned}
$$

This theorem can be readily generalized to the case where $P: M \rightarrow M_{n-k}$ is a projection on an $n-k$ dimensional sublattice. In particular for $n=4$ and $k=2$ we can use it to study elliptic fibrations of 3-folds. The duality of projections and intersections is most easily visualised with the help of a simple example: Fig. 1 shows a dual pair of reflexive triangles. The first triangle has reflexive intersections along both coordinate axes, corresponding to reflexive projections on the coordinate axes in the second triangle (along the direction of the other axis). The second triangle has only one reflexive intersection (along the vertical axis), corresponding to the projection on the vertical axis (along the horizontal axis) in the first triangle.

Let us now convince ourselves that reflexive polyhedra fulfilling the criteria of the theorem correspond to spaces allowing Calabi-Yau hypersurfaces that are fibrations whose generic fibers are lower dimensional Calabi-Yau hypersurfaces. Let us repeat our ingredients: In the $M$ lattice we have a distinguished direction with integer generator $e_{n}$ (as in the proof of the theorem) defining a projection $P$ that projects our polyhedron $\Delta$ to a reflexive polyhedron $\Delta_{n-1}$. In the $N$ lattice we have a distinguished hyperplane

$$
H=\left\{y \in N:\left\langle y, e_{n}\right\rangle=0\right\}
$$

whose intersection with $\Delta^{*}$ is $\Delta_{n-1}^{*}$. 
In the gluing approach we have the following picture: Depending on whether we consider $\Delta_{n-1}^{*}$ as a polytope in $N$ or in $N_{n-1}=N \cap H$, it defines $n$ or $n-1$ dimensional varieties related by

$$
V_{\Delta_{n-1}^{*}, N} \simeq V_{\Delta_{n-1}^{*}, N_{n-1}} \times \mathbb{C}^{*}
$$

(see, for example, pages 5,6 of [15]). Therefore $V_{\Delta}$ can be considered as a compactification of $V_{\Delta_{n-1}^{*}, N_{n-1}} \times \mathbb{C}^{*}$, giving a first hint in the desired direction.

A clearer picture emerges in the holomorphic quotient approach: The integer generators $v_{1}, \cdots, v_{N}$ of the one dimensional cones are just the integer points of $\Delta^{*}$. We may split this set into $\left\{v_{1}, \cdots, v_{N^{\prime}}\right\}$ (corresponding to points of $\Delta_{n-1}^{*}$ ) and the remaining set $\left\{v_{N^{\prime}+1}, \cdots, v_{N}\right\}$. The latter set may again be decomposed, namely into the set of points 'above' and the set of points 'below' $\Delta_{n-1}^{*}$. Then we may choose our linear relations such that we have $N^{\prime}-(n-1)$ relations involving only $v_{1}, \cdots, v_{N^{\prime}}$ and $N-N^{\prime}-1$ relations involving all of the $v_{i}$. Similarly we may split our set $Z_{\Sigma_{n}}$ as $Z_{\Sigma_{n}}=\left(Z_{\Sigma_{n-1}} \times \mathbb{C}^{N-N^{\prime}}\right) \cup Z_{\text {diff }}$. With a slight abuse of notation, the embedding toric variety is then given by

$$
\left\{\left[\left[\left(\mathbb{C}^{N^{\prime}} \backslash Z_{\Sigma_{n-1}}\right) / \mathbb{C}^{* N^{\prime}+1-n}\right] \times \mathbb{C}^{N-N^{\prime}}\right] \backslash Z_{\text {diff }}\right\} / \mathbb{C}^{* N-N^{\prime}-1}
$$

The direction of the projection $P$ determines a ray in the $M$ lattice whose integer generator $e_{n}$ corresponds to a Laurent monomial

$$
q\left(z_{N^{\prime}+1}, \cdots, z_{N}\right)=\prod_{j=1}^{N} z_{j}^{\left\langle v_{j}, e_{n}\right\rangle}=\prod_{j=N^{\prime}+1}^{N} z_{j}^{\left\langle v_{j}, e_{n}\right\rangle} .
$$

We will now argue that the value of $q$ determines a point in our base space $\mathbb{P}^{1}$. A short glance at eq. (1) shows us that $q$ is invariant under the equivalence relations. It takes the value 0 if one of the $z_{j}$ corresponding to points above $\Delta_{n-1}^{*}$ is 0 , and infinity if one of the $z_{j}$ corresponding to points below $\Delta_{n-1}^{*}$ is 0 . If we choose our maximal triangulation of $\Delta^{*}$ in such a way that it contains a maximal triangulation of $\Delta_{n-1}^{*}$, the rules for constructing $Z_{\Sigma}$ tell us that these two cases cannot occur simultaneously. Thus we have indeed a well-defined (and obviously surjective) map from $V_{\Sigma}$ to $\mathbb{P}^{1}$. The quasihomogeneous polynomial of eq. (2) may be seen as a polynomial in $z_{1}, \cdots, z_{N^{\prime}}$ (quasihomogeneous with respect to the first $N^{\prime}-n+1$ relations) with coefficients that are polynomials in $z_{N^{\prime}+1}, \cdots, z_{N}$. After fixing a point in our base space $\mathbb{P}^{1}$ we may then proceed to determine the structure of the fiber. In particular, if the point in the base space lies in $\mathbb{C}^{*} \subset \mathbb{P}^{1}$, we may use the second set of relations to eliminate all coordinates $z_{N^{\prime}+1}, \cdots, z_{N}$, thus obtaining the fiber as the zero locus of a quasihomogeneous polynomial in the space determined by $\Delta_{n-1}$. This shows us that the generic fiber in this construction is an $n-2$ dimensional CY manifold. A similar result is obtained at the level of embedding varieties. Take a point where $q \neq 0$ is finite. Since $z_{N^{\prime}+1}, \cdots, z_{N}$ are all different from zero there are no constraints on the rest of the degrees of freedom coming from $Z_{\text {diff }}$ which thus span the whole $V_{\Sigma_{n-1}}$.

How this works is best understood with the help of some examples. The following two examples are based on 3 dimensional polyhedra corresponding to elliptic fibrations of K3 manifolds, because they can be visualised more easily. Nevertheless they exhibit all of the features that are essential also in the 4 dimensional case. 


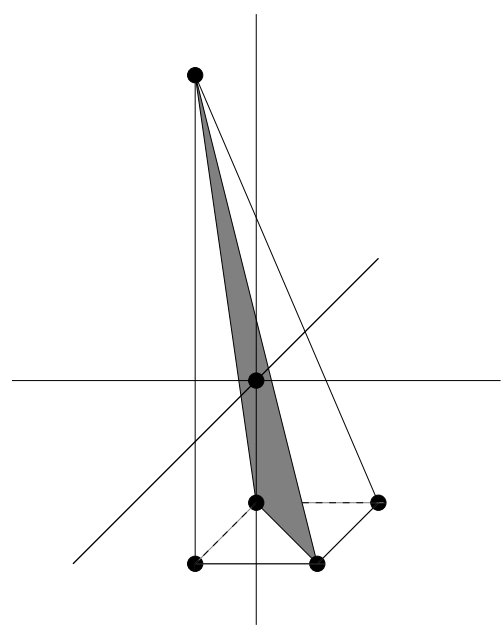

Figure 2: The dual polytope $\Delta^{*}$ of Example 1

Example 1: Take as $\Delta^{*}$ the pyramid with peak at $v_{1}=(-1,-1,2)$ and base the square whose vertices are $v_{2}=(0,0,-1), \quad v_{3}=(1,1,-1), \quad v_{4}=(0,1,-1)$ and $v_{5}=(1,0,-1)$. It is easily checked that there are no other integer points (except the origin). We have two linear relations: $v_{1}+v_{2}+v_{3}=0$ and $v_{1}+v_{4}+v_{5}=0$. If we triangulate the base of the pyramid along the diagonal $\overline{v_{2} v_{3}}$, we get $Z_{\Sigma}=\left\{z_{1}=z_{2}=z_{3}=0\right\} \cup\left\{z_{4}=z_{5}=0\right\}$. With these data we can describe a 2-dimensional Calabi-Yau space (a K3 surface) as a codimension 1 surface in $\left(\mathbb{C}^{5} \backslash Z_{\Sigma}\right) /\left(\mathbb{C}^{*}\right)^{2}$, where the $\left(\mathbb{C}^{*}\right)^{2}$ action is determined by

$$
\left(z_{1}, z_{2}, z_{3}, z_{4}, z_{5}\right) \sim\left(\lambda \mu z_{1}, \lambda z_{2}, \lambda z_{3}, \mu z_{4}, \mu z_{5}\right)
$$

The polynomial describing the surface is given by

$$
z_{1}^{3}+z_{1}^{2}\left(z_{2} p_{1}^{(1)}+z_{3} p_{1}^{(2)}\right)+z_{1}\left(z_{2}^{2} p_{2}^{(1)}+z_{2} z_{3} p_{2}^{(2)}+z_{3}^{2} p_{2}^{(3)}\right)+z_{2}^{3} p_{3}^{(1)}+z_{2}^{2} z_{3} p_{3}^{(2)}+z_{2} z_{3}^{2} p_{3}^{(3)}+z_{3}^{3} p_{3}^{(4)},
$$

where $p_{1}^{(.)}, p_{2}^{(.)}, p_{3}^{(.)}$are linear, quadratic and cubic polynomials in $z_{4}$ and $z_{5}$, respectively. Clearly the whole polynomial is homogeneous of degree 3 both in $\lambda$ and in $\mu$. Our base space is $\mathbb{P}^{1}$ with homogeneous coordinates $\left(z_{4}: z_{5}\right)$. In the coordinate patch $z_{4} \neq 0\left(z_{5} \neq 0\right)$ we may use $\mu$ to set $z_{4}\left(z_{5}\right)$ to 1 . Fixing a point in the base space amounts to fixing the values of the $p_{i}^{(.)}$. The generic fiber is an elliptic curve determined by a cubic equation in $\mathbb{P}^{2}$ with homogeneous coordinates $\left(z_{1}: z_{2}: z_{3}\right)$.

Example 2: Again we consider $N \simeq \mathbb{Z}^{3}$. The polyhedron $\Delta_{2}^{*}$ corresponding to the fiber (the shaded area in Fig.3) is determined by the vertices $v_{1}=(1,0,0), v_{2}=(0,1,0), v_{3}=(-1,-1,0)$, and the polyhedron $\Delta_{3}^{*}$ has in addition two 'upper' points $v_{4}=(0,0,1), v_{5}=(-1,-1,1)$ and one 'lower'point $v_{6}=(0,0,-1)$.

We have the linear relations $v_{1}+v_{2}+v_{3}=0, v_{4}+v_{6}=0$ and $v_{1}+v_{2}+v_{5}+v_{6}=0 . \Delta_{3}^{*}$ has only triangular faces and

$Z_{\Sigma}=\left\{z_{1}=z_{2}=z_{3}=0\right\} \cup\left\{z_{1}=z_{2}=z_{5}=0\right\} \cup\left\{z_{3}=z_{4}=0\right\} \cup\left\{z_{4}=z_{6}=0\right\} \cup\left\{z_{5}=z_{6}=0\right\}$. 


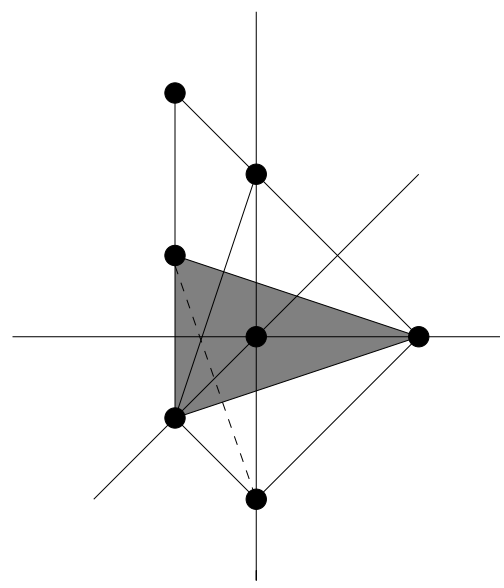

Figure 3: The dual polytope $\Delta_{3}^{*}$ of Example 2

$V_{\Sigma}$ is the space $\left(\mathbb{C}^{6} \backslash Z_{\Sigma}\right) /\left(\mathbb{C}^{*}\right)^{3}$ with the $\left(\mathbb{C}^{*}\right)^{3}$ action given by

$$
\left(z_{1}, z_{2}, z_{3}, z_{4}, z_{5}, z_{6}\right) \sim\left(\lambda \nu z_{1}, \lambda \nu z_{2}, \lambda z_{3}, \mu z_{4}, \nu z_{5}, \mu \nu z_{6}\right)
$$

We can describe a K3 surface in this space by an equation of the type

$$
z_{1}^{3} p_{1}^{(1)}+z_{1}^{2} z_{2} p_{1}^{(2)}+z_{1} z_{2}^{2} p_{1}^{(3)}+z_{2}^{3} p_{1}^{(4)}+z_{3}\left(z_{1}^{2} p_{2}^{(1)}+z_{1} z_{2} p_{2}^{(2)}+z_{2}^{2} p_{2}^{(3)}\right)+z_{3}^{2}\left(z_{1} p_{3}^{(1)}+z_{2} p_{3}^{(2)}\right)+z_{3}^{3} p_{4}=0
$$

where the $p_{i}^{(\cdot)}$ are polynomials in $z_{4}, z_{5}, z_{6}$ with bidegree $(2, i)$ with respect to $(\mu, \nu)$. The base space $\mathbb{P}^{1}$ is $\left(z_{4} z_{5}: z_{6}\right)$. In the patch where $z_{4} z_{5} \neq 0$ we may use $\mu$ and $\nu$ to set $z_{4}$ and $z_{5}$ to 1 , thus taking $z_{6}$ as the variable parametrizing the $\mathbb{P}^{1}$. Again the generic fiber is an elliptic curve given by a cubic equation in $\mathbb{P}^{2}$ with homogeneous coordinates $\left(z_{1}: z_{2}: z_{3}\right)$. At the point $z_{4} z_{5}=0$, however, the fiber becomes degenerate: If $z_{4}=0$ then $z_{3} \neq 0$ and $z_{6} \neq 0$. With suitable redefinitions of $\lambda, \mu, \nu$ and partial fixing of the variables we obtain the $z_{4}=0$ part of the fiber as the zero locus of a quadratic equation in a $\mathbb{P}^{2}$ described by

$$
\left(z_{1}, z_{2}, 1,0, z_{5}, 1\right) \sim\left(\nu z_{1}, \nu z_{2}, 1,0, \nu z_{5}, 1\right)
$$

i.e. the $z_{4}=0$ part of the singular fiber is a double cover of $\mathbb{P}^{1}$. At $z_{5}=0$ we have $\left(z_{1}, z_{2}\right) \neq$ $(0,0)$. After suitable redefinitions of $\lambda, \mu, \nu$ we get an equation of the type

$$
p_{3}\left(z_{1}, z_{2}\right) z_{4}+p_{2}\left(z_{1}, z_{2}\right) z_{3}=0
$$

in a space described by

$$
\left(z_{1}, z_{2}, z_{3}, z_{4}, 0,1\right) \sim\left(\alpha z_{1}, \alpha z_{2}, \alpha \beta z_{3}, \beta z_{4}, 0,1\right)
$$

This space may be projected to a $\mathbb{P}^{1}$ described by $\left(z_{1}: z_{2}\right)$. Then the above equation determines $z_{3}$ and $z_{4}$ uniquely up to $\beta$ equivalence at each point $\left(z_{1}: z_{2}\right)$, so the $z_{5}=0$ part of the fiber over $z_{4} z_{5}=0$ is just $\mathbb{P}^{1}$. The two parts of the singular fiber intersect at the two points corresponding to the zero locus of a quadratic equation in $\mathbb{P}^{1}$ :

$$
\left(z_{1}, z_{2}, 1,0,0,1\right) \sim\left(\alpha z_{1}, \alpha z_{2}, 1,0,0,1\right)
$$


Returning to our general discussion, we may ask whether our projection $P \Delta$ in the $M$ lattice is isomorphic to the intersection of $\Delta$ with some lattice hyperplane $H$. If $H$ intersects the interior of $\Delta$, then the only possibility for $\Delta \cap H$ to have an interior point is that this point is the interior point of $\Delta$. If $H$ does not intersect the interior of $\Delta$, then the requirement that $\Delta \cap H$ is an $n-1$ dimensional polyhedron tells us that $H$ must in fact be a bounding hyperplane, i.e. that $\Delta \cap H$ is a facet of $\Delta$. Summing up, there are three possibilities:

1. There is no hyperplane $H$ such that $P \Delta$ is isomorphic to $H \cap \Delta$;

2. $P \Delta$ is isomorphic to $H \cap \Delta$, where $H$ is a hyperplane through the origin;

3. $P \Delta$ is isomorphic to a facet of $\Delta$.

Of course cases 2 and 3 are not mutually exclusive. An example is provided by the first triangle of Fig.1, with $P$ the projection along the horizontal axis. There are also examples of polyhedra allowing different projections to lower dimensional polyhedra, corresponding to different cases. For the second triangle of Fig.1, the projection along the horizontal axis corresponds to case 2 whereas the projection along the vertical axis corresponds to case 3. Case 2 is particularly pretty because there not only the CY-manifold we are considering but also its mirror have the structure of a fibration. It is realised by the projections along the horizontal axes in Fig. 1. Case 1 is realised, for example, by the triangle with vertices $(1,0),(0,1)$ and $(-1,-1)$ (the dual of the Newton polyhedron of $\left.\mathbb{P}^{2}\right)$. Here the projections along both coordinate directions and along the $(1,1)$-direction are reflexive, but no intersection is.

Let us now consider possible strategies for looking for torically realised K3 fibrations.

Of course a complete analysis is possible by considering all 3 dimensional hyperplanes in the $N$ lattice that are linearly spanned by points of $\Delta^{*}$, one of which is the interior point. To this end, we would have to analyse $\left(\begin{array}{c}l-1 \\ 3\end{array}\right)$ hyperplanes for each polyhedron, where $l$ is the number of points in $\Delta$. We did not attempt to do that.

A more modest approach is to search for projections corresponding to case 3 . Hosono, Lian and Yau [12] have analysed the 7,555 CY-hypersurfaces corresponding to transverse polynomials in weighted projective spaces with respect to a specialisation of this case, where the reflexivity of the facet can be read off from the weights. In the present work we extend their results in two directions: On the one hand we consider not only the 7,555 old models, but the 184,026 models of [8], and on the other hand we analyse the geometry of the faces instead of properties of weights, thereby finding all fibrations corresponding to facets.

In fact, there is even more that we can learn by considering facets of $\Delta$ : Unless the projection $P$ is parallel to some facet $\theta$, any interior point of $\theta$ will be mapped to an interior point of $P \Delta$. In particular, this implies the following: If $\theta$ has more than one interior point, $P \Delta$ can only be reflexive if $P$ is parallel to $\theta$, and if $\theta$ has exactly one interior point and $P \Delta$ is reflexive, then either $P$ is parallel to $\theta$ or the interior point of $\theta$ is mapped to the interior point of $P \Delta$. If $\Delta$ has enough facets with interior points, these considerations are sufficient to determine all reflexive projections. 
In the following section we will consider maximal Newton polyhedra [7] corresponding to single weight systems (classified in [8]) and show how special properties of these polyhedra make the application of these strategies particularly simple.

\section{Maximal Newton polyhedra}

In ref. [7], an algorithm for the classification of reflexive polyhedra was presented. The key to this algorithm is the fact that any reflexive polyhedron is a subpolyhedron of a polytope defined by a weight system or a combination of weight systems, in the following way: A weight system is just a collection of positive rational numbers $q_{i}=w_{i} / d$ with $\sum q_{i}=1\left(\sum w_{i}=d\right)$. In the present work we consider only the case of a single weight system with 5 weights. $\left(w_{1}, \cdots, w_{5}\right)$ defines a sublattice $\Gamma^{4}$ of the lattice $\Gamma^{5} \simeq \mathbb{Z}^{5}$ by

$$
\Gamma^{4}=\left\{\left(x^{i}\right) \in \Gamma^{5}: \sum w_{i} x^{i}=d\right\}
$$

If we also consider the real extensions $\Gamma_{\mathbb{R}}^{5}$ and $\Gamma_{\mathbb{R}}^{4}$, we may define the simplex $Q$ (whose vertices are generically rational) as the intersection of $\Gamma_{\mathbb{R}}^{4}$ with the positive hyperoctant in $\Gamma_{\mathbb{R}}^{5}$ :

$$
Q=\left\{\left(x^{i}\right) \in \Gamma_{\mathbb{R}}^{4}: x^{i} \geq 0 \quad \forall i\right\}
$$

and the maximal Newton polyhedron (MNP) $\Delta_{\max }$ as the set of integer points in $Q: \Delta_{\max }=$ $Q \cap \Gamma^{4}$. It is easy to see that the point $\mathbf{1}=(1, \cdots, 1)$ is the only integer point in the interior of $Q$, and that its integer distance to any of the hyperplanes $x^{i}=0$ bounding $Q$ is 1 . If 1 is also in the interior of $\Delta_{\max }$ (which we assume henceforth), we say that the weight system $\left(w_{1}, \cdots, w_{5}\right)$ has the 'interior point property'. These weight systems, which play a crucial role for the algorithm of [7], were classified in [8]. In particular, in the case of 4 dimensions considered here, $\Delta_{\max }$ is reflexive by another result of [8]. The $M$ lattice is identified with $\Gamma_{4}$, with $\mathbf{0}_{M}$ corresponding to 1 .

It is tempting to identify spaces defined by an MNP with the weighted projective spaces defined by the weight system. A weighted projective space, however, is a toric variety, determined by a fan with $n+1$ one-dimensional cones. In our language, these are the cones over the vertices of the integer (but not reflexive) simplex $Q^{*}$. One can define a weighted projective space with any set of weights, but the resulting variety is usually very ill behaved, whereas the singularities of spaces defined by maximal triangulations of reflexive polyhedra have codimension $\geq 4$ [6]. What we consider are the varieties defined by the corresponding MNP's, which are blowups of weighted projective spaces, because $\Delta_{\max } \subset Q$ implies $Q^{*} \subset \Delta_{\text {max }}^{*}$. A special case occurs when the vertices of $Q^{*}$ are not just points, but also vertices of $\Delta^{*}$. Then, by duality, the hyperplanes dual to these points (which are just the coordinate hyperplanes $x^{i}=0$ which carry the facets of the simplex $Q$ ) must also carry facets of $\Delta$. Hence vertices of $Q^{*}$ are vertices of $\Delta^{*}$ if and only if the hyperplanes $x^{i}=0$ are affinely spanned by points of $\Delta$. In this case we say that a weight system has the 'span property'. The weight systems with both the 'interior point' and the 'span property' are the ones that are relevant for the classification scheme of [7]. 
As we have seen in the previous section, interior points of facets are the key ingredients for our strategies for the search for reflexive projections of reflexive polyhedra. The following lemmata will provide useful tools for identifying interior points of facets of MNP's. They all refer to MNP's defined by a single weight system.

Lemma 1: An integer interior point of a facet $x^{k}=0$ of $Q$ defines a projection $P: \Gamma_{\mathbb{R}}^{4} \rightarrow$ $\Gamma_{\mathbb{R}}^{4} \cap\left\{x^{k}=0\right\}$ such that $P \Delta_{\max }=\Delta_{\max } \cap\left\{x^{k}=0\right\}$.

Proof: An integer interior point of a facet $x^{k}=0$ of $Q$ must have coordinates $x^{k}=0$ and $x^{i}=1+y^{i}, y^{i} \geq 0$ for $i \neq k$ (if one of the $x^{i}$ with $i \neq k$ were zero, our point would be at the boundary of the facet $\left.x^{k}=0\right)$. By comparison with the interior point $(1, \cdots, 1)$ of $\Delta_{\max }$ we see that $\sum_{i \neq k} w_{i} y^{i}=w_{k}$. Then, for $\left(x^{1}, \cdots, x^{5}\right) \in \Gamma_{\mathbb{R}}^{4}, P$ is defined by $x^{k} \rightarrow 0, x^{i} \rightarrow x^{i}+y^{i} x^{k}$ for $i \neq k$. Obviously $\Delta_{\max } \cap\left\{x^{k}=0\right\} \subseteq P \Delta_{\max }$, and the maximality assumption on $\Delta_{\max }$ also ensures $P \Delta_{\max } \subseteq \Delta_{\max } \cap\left\{x^{k}=0\right\}$.

Lemma 2: An integer interior point of $Q \cap\left\{x^{k}=0\right\}$ is also an interior point of $\Delta_{\max } \cap\left\{x^{k}=0\right\}$. Proof: An integer interior point of $Q \cap\left\{x^{k}=0\right\}$ is the image of $(1, \cdots, 1)$ under the projection $P$ of Lemma 1. If it were at the boundary of $\Delta_{\max } \cap\left\{x^{k}=0\right\}=P \Delta_{\max }$, then $(1, \cdots, 1)$ would be at the boundary of $\Delta_{\max }$.

Lemma 3: (1) Facets of $\Delta_{\max }$ that do not correspond to hyperplanes of the type $x^{k}=0$ have no interior points.

(2) Interior points of a facet of $\Delta_{\max }$ defined by $x^{k}=0$ are in one-to-one correspondence with partitions of $w_{k}$ by $\left\{w_{i}: i \neq k\right\}$.

Proof: (1) Such a point would have to be in the interior of $Q$, but the integer interior point of $Q$ is unique and not on a facet.

(2) Partitions of $w_{k}$ by $\left\{w_{i}: i \neq k\right\}$ define projections as in the proof of Lemma 2. Projections of $(1, \cdots, 1)$ are integer interior points of $Q \cap\left\{x^{k}=0\right\}$ and (by Lemma 1) of $\Delta_{\max } \cap\left\{x^{k}=0\right\}$. There is a one-to-one correspondence between partitions of $w_{k}$ and interior points of $\Delta_{\max } \cap\left\{x^{k}=0\right\}$ defined by the corresponding projections.

Lemma 3 is the key to our strategies for identifying reflexive projections:

Searching for reflexive facets, we simply look for weights that have unique partitions by the other weights. By Lemma 1 we can be sure that such a facet corresponds to a projection. For 3 dimensional polyhedra (weight systems with 4 weights) Lemma 3 would even guarantee reflexivity of the facet, because any polygon with a single interior point is reflexive. In the present case we still have to check for reflexivity of the facet, and indeed it turns out that there are many cases where a facet has a single interior point without being reflexive. If, for example, $w_{1}$ has the unique partition $w_{1}=\sum_{i>1} w_{i} y^{i}$, then the vector $e_{n}$ determining our projection $P$ is given by $\left(-1, y_{2}, \cdots, y_{5}\right)$.

On the other hand, if we have three facets ( points, then any projection that does not correspond to one of these facets must be parallel to each of them. Thus it must be parallel to the edge $x^{1}=x^{2}=x^{3}=0$ of $Q$, connecting the vertices $\left(0,0,0, d / w_{4}, 0\right)$ and $\left(0,0,0,0, d / w_{5}\right)$ of $Q$. Denoting by $g$ the greatest common divisor of $w_{4}$ and $w_{5}, e_{n}=\left(0,0,0, w_{5} / g,-w_{4} / g\right)$. With four or five facets with interior points (this 
happens, in particular, if we have one or two weights equal to 1), projections not corresponding to facets are excluded.

\section{$5 \quad$ Results}

In order to find K3 fibrations we pursued the two different strategies described in the previous sections. In the search for K3 facets we first searched for weights that have a unique partition in the remaining weights, which is a necessary condition for a unique interior point and hence for reflexivity. The results are listed in Table 1 for the complete list of reflexive weights and for the much smaller list of spanning weights.

\begin{tabular}{||c||c|c|c|c||c||}
\hline \hline weights & no UP weight & 1 UP weight & 2 UP weights & 3 UP weights & total \\
\hline \hline reflexive & 43,988 & 133,386 & 6,571 & 81 & 184,026 \\
\hline spanning & 9,939 & 26,400 & 2,361 & 27 & 38,727 \\
\hline \hline
\end{tabular}

Table 1: Numbers of weights with unique partitions

If we keep only those weights whose corresponding facets actually are reflexive then the numbers reduce to the numbers of K3 fibrations that arise as projections onto facets as given in Table 2. It turns out that all of the 81 polytopes where 3 weights have a unique partition have at most 1 reflexive facet. An example of this type is $(3,4,6,7,8 ; 28)$, where 6,7 and 8 have unique partitions while only the facet corresponding to the weight 7 is reflexive.

\begin{tabular}{||c||c|c|c||c||}
\hline \hline weights & no K3 facet & $1 \mathrm{~K} 3$ facet & 2 K3 facets & total \\
\hline \hline reflexive & 76,460 & 104,036 & 3,530 & 184,026 \\
\hline spanning & 19,410 & 18,356 & 961 & 38,727 \\
\hline \hline
\end{tabular}

Table 2: Numbers of K3 facets

In both tables partitions of equal weights are counted only once because the corresponding projections onto facets are along the same direction $e_{n}= \pm(1,-1,0, \ldots)$ and thus define the same fibration (regardless of the fact that there are two reflexive facets for each such pair of equal weights). This is always the case, for example, if 2 and only 2 weights are equal to 1 (this type of example has been used extensively in the literature). A nice case with 2 pairs of equal weights is $(2,2,3,3,4 ; 14)$, where 2 and 3 have unique partitions, so there are 4 reflexive facets but only 2 inequivalent K3 fibrations (see Table 6 below).

Our alternative search method, which is based on the constraints on directions of projection due to facets with interior points, is not applicable in $55 \%$ of all cases although about half of these even have projections onto K3 facets. In the remaining cases it allows, however, for a complete analysis, providing many examples with up to 3 different K3 projections and 


\begin{tabular}{||c||c|c|c||c||}
\hline \hline & $\# F=0$ & $\# F=1$ & $\# F=2$ & total \\
\hline \hline$\# \Pi=?$ & 54,195 & 45,792 & 1,508 & 101,495 \\
\hline$\# \Pi=0$ & 5,130 & & & 5,130 \\
\hline$\# \Pi=1$ & 17,135 & 20,080 & & 37,215 \\
\hline$\# \Pi=2$ & 0 & 38,164 & 1,011 & 39,175 \\
\hline$\# \Pi=3$ & 0 & 0 & 1,011 & 1,011 \\
\hline \hline total & 76,460 & 104,036 & 3,530 & 184,026 \\
\hline \hline
\end{tabular}

Table 3: Numbers of K3 projections (\#П) and K3 facets $(\# F)$

\begin{tabular}{||c||c|c|c||c||}
\hline \hline TS & $\# F=0$ & $\# F=1$ & $\# F=2$ & total \\
\hline \hline$\# \Pi=?$ & 703 & 299 & 12 & 1014 \\
\hline$\# \Pi=0$ & 507 & & & 507 \\
\hline$\# \Pi=1$ & 697 & 1,471 & & 2,168 \\
\hline$\# \Pi=2$ & 0 & 383 & 88 & 471 \\
\hline$\# \Pi=3$ & 0 & 0 & 9 & 9 \\
\hline \hline total & 1,907 & 2,153 & 109 & 4,169 \\
\hline \hline
\end{tabular}

Table 4a: Transverse spanning weights

\begin{tabular}{||c||c|c|c||c||}
\hline \hline$-\mathrm{S}$ & $\# F=0$ & $\# F=1$ & $\# F=2$ & total \\
\hline \hline$\# \Pi=?$ & 10,222 & 3,804 & 297 & 14,323 \\
\hline$\# \Pi=0$ & 2,258 & & & 2,258 \\
\hline$\# \Pi=1$ & 5,023 & 6,692 & & 11,715 \\
\hline$\# \Pi=2$ & 0 & 5,707 & 356 & 6,063 \\
\hline$\# \Pi=3$ & 0 & 0 & 199 & 199 \\
\hline \hline total & 17,503 & 16,203 & 852 & 34,558 \\
\hline \hline
\end{tabular}

Table 4c: Non-transverse spanning weights

\begin{tabular}{||c||c|c|c||c||}
\hline \hline $\mathrm{T}-$ & $\# F=0$ & $\# F=1$ & $\# F=2$ & total \\
\hline \hline$\# \Pi=?$ & 859 & 622 & 39 & 1,520 \\
\hline$\# \Pi=0$ & 116 & & & 116 \\
\hline$\# \Pi=1$ & 536 & 586 & & 1,122 \\
\hline$\# \Pi=2$ & 0 & 577 & 27 & 604 \\
\hline$\# \Pi=3$ & 0 & 0 & 24 & 24 \\
\hline \hline total & 1,511 & 1,785 & 90 & 3,386 \\
\hline \hline
\end{tabular}

Table 4b: Transverse non-spanning weights

\begin{tabular}{||c||c|c|c||c||}
\hline \hline-- & $\# F=0$ & $\# F=1$ & $\# F=2$ & total \\
\hline \hline$\# \Pi=?$ & 42,411 & 41,067 & 1160 & 84,638 \\
\hline$\# \Pi=0$ & 2,249 & & & 2,249 \\
\hline$\# \Pi=1$ & 10,879 & 11,331 & & 22,210 \\
\hline$\# \Pi=2$ & 0 & 31,497 & 540 & 32,037 \\
\hline$\# \Pi=3$ & 0 & 0 & 779 & 779 \\
\hline \hline total & 55,539 & 83,895 & 2,479 & 141,913 \\
\hline \hline
\end{tabular}

Table 4d: Non-transverse non-spanning weights

a powerful consistency check. In particular, for 5,130 weights we have shown that the corresponding Newton polytopes do not admit any reflexive projection. It is interesting to compare the results of our two approaches in some detail, and this is done by the compilations in the following tables, where we first give the overall statistics in Table 3, and then list the respective numbers for the weights that do $(\mathrm{T})$ or do not $(-)$ admit transverse polynomials and for which the Newton polytopes do $(\mathrm{S})$ or do not $(-)$ span all coordinate hyperplanes in Table 4 . It turns out that the number of toric fibrations goes up to 3. Actually that number can go up to at least 5, as one can see from the example of the mirror of the quintic threefold for which any projection along a line from the origin to one of the 5 vertices is reflexive (since none of the facets has an interior point, however, our algorithms do not find these fibrations). 
In Table 5 we give the beginning and the end of our complete list of results which has 184,026 lines and is available via $\mathrm{WWW}^{5}$ or by e-mail from the authors. Weights with unique partitions in terms of the other weights such that the facets carried by the corresponding coordinate hyperplanes are reflexive are typed in bold face (if a weight occurs twice it is only marked once since the pair defines only a single fibration; see above). We also list the Hodge numbers $h_{11}$ and $h_{21}$ and the numbers $P$ and $V$ of points and vertices for the MNP and its dual, and also indicate the transversality and spanning properties of the weights.

In Table 6 we give the same information for a number of examples that illustrate the phenomena that can occur. The first line is an example of a weight combination whose MNP is the mirror of the quintic, i.e. a simplex with 6 points such that all vertices have distance 5 from the opposite facet. This polytope has 5 reflexive projections, but none is onto a facet and it also cannot be analysed with our second approach since none of the facets has an interior point. The second example features the maximal number of vertices of $\Delta$, which is $18 ; \Delta^{*}$ can have up to 21 vertices, which occurs twice. The number of points goes up to 680 both for $\Delta$ (this number occurs for the Fermat weights in the next line of Table 6 ) and $\Delta^{*}$ (apparently the dual of the Fermat simplex, represented many times by different weight systems, for example at the end of Table 5). The remaining lines give examples (of minimal degree) for all combinations of transversality and spanning properties, general projections and projections onto facets.

It is straightforward to apply our methods also to the well known $95 \mathrm{~K} 3$ weights, this time producing elliptic fibrations. Since all 2D polytopes with one interior point are automatically reflexive, no reflexivity check is required and the search for 'elliptic' facets can be done by hand. The results are listed in Table 7 , where the (up to 2) weights with unique partition are again in bold face. With our alternative search algorithm we again find up to 3 fibrations per Newton polytope, so that we find a total of 110 ellipitic fibrations. ${ }^{6}$ The statistics are given in Table 8.

We also computed the Hodge numbers and compared and combined them with the complete results that exist for weighted projective spaces [4,5] and abelian orbifolds thereof [19] in Table 9. A pronounced feature is the absence of mirror symmetry, which is already familiar from the list of transversal weights. The well known plot Fig.4 becomes much denser, but otherwise does not change shape. In our context it does not make sense any longer to omit the mirror spectra since they are produced by the dual polytopes (which, however, cannot be MNPs for a single weight system whenever the spectrum does not occur in our original list). Counting all spectra of MNPs and their duals we thus get an increase in the known Calabi-Yau spectra by more than a factor of 3. Inclusion of the abelian orbifold spectra for the transversal weights, which have been analysed completely in [19], only adds 173 new spectra to those for reflexive MNPs of single weight systems. If we mirror-symmetrize by hand, as is appropriate in the toric framework (all abelian orbifolds correspond to MNPs on sublattices, which are reflexive because of the results of [8]), this number even goes down to 95. This can be interpreted as an indication that our list of spectra already might be fairly complete.

\footnotetext{
5 The URL is http://tph.tuwien.ac.at/ kreuzer/CY

6 The algorithm of [12] yields 18, with 1 double fibration.
} 


\begin{tabular}{|c|c|c|c|c|c|c|c|c|c|c|c|c|c|c|}
\hline$d$ & $w_{1}$ & $w_{2}$ & $w_{3}$ & $w_{4}$ & $w_{5}$ & $\mathrm{TS}$ & $h_{11}$ & $h_{12}$ & $\# P$ & $\# V$ & $\# \bar{P}$ & $\# \bar{V}$ & $\Pi$ & $\mathrm{F}$ \\
\hline 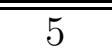 & 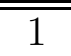 & 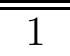 & 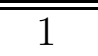 & 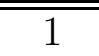 & 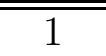 & 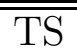 & $\bar{~} 1$ & $\bar{~} 101$ & $\bar{~} 126$ & 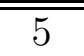 & $\bar{~} 6$ & $\bar{~} 5$ & 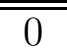 & 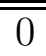 \\
\hline 6 & 1 & 1 & 1 & 1 & 2 & TS & 1 & 103 & 130 & 5 & 6 & 5 & 0 & 0 \\
\hline 7 & 1 & 1 & 1 & 1 & 3 & $\mathrm{TS}$ & 2 & 122 & 159 & 8 & 7 & 6 & 0 & 0 \\
\hline 7 & 1 & 1 & 1 & 2 & 2 & TS & 2 & 95 & 120 & 9 & 7 & 6 & 0 & 0 \\
\hline 8 & 1 & 1 & 1 & 1 & 4 & TS & 1 & 149 & 201 & 5 & 7 & 5 & 0 & 0 \\
\hline 8 & 1 & 1 & 1 & 2 & 3 & TS & 2 & 106 & 136 & 8 & 7 & 6 & 0 & 0 \\
\hline 8 & 1 & 1 & 2 & 2 & 2 & $\mathrm{TS}$ & 2 & 86 & 105 & 5 & 7 & 5 & 1 & 1 \\
\hline 9 & 1 & 1 & 1 & 2 & 4 & TS & 3 & 123 & 162 & 9 & 8 & 6 & 0 & 0 \\
\hline 9 & 1 & 1 & 1 & 3 & 3 & TS & 4 & 112 & 145 & 5 & 7 & 5 & 0 & 0 \\
\hline 9 & 1 & 1 & 2 & 2 & 3 & $\mathrm{TS}$ & 2 & 86 & 109 & 9 & 7 & 6 & 0 & 0 \\
\hline 10 & 1 & 1 & 1 & 2 & 5 & $\mathrm{TS}$ & 1 & 145 & 196 & 5 & 7 & 5 & 0 & 0 \\
\hline 10 & 1 & 1 & 1 & 3 & 4 & $-\mathrm{S}$ & 4 & 126 & 165 & 10 & 9 & 7 & 0 & 0 \\
\hline 10 & 1 & 1 & 2 & 2 & 4 & TS & 3 & 99 & 126 & 8 & 8 & 6 & 1 & 1 \\
\hline 10 & 1 & 1 & 2 & 3 & 3 & $\mathrm{TS}$ & 3 & 87 & 111 & 9 & 8 & 6 & 0 & 0 \\
\hline 10 & 1 & 2 & 2 & 2 & 3 & TS & 3 & 75 & 87 & 8 & 8 & 6 & 0 & 0 \\
\hline 11 & 1 & 1 & 1 & 3 & 5 & TS & 4 & 144 & 192 & 10 & 9 & 7 & 0 & 0 \\
\hline 11 & 1 & 1 & 2 & 2 & 5 & TS & 4 & 109 & 144 & 10 & 9 & 7 & 1 & 1 \\
\hline 11 & 1 & 1 & 2 & 3 & 4 & $\mathrm{TS}$ & 4 & 94 & 121 & 13 & 9 & 8 & 0 & 0 \\
\hline 11 & 1 & 2 & 2 & 3 & 3 & $\mathrm{TS}$ & 4 & 64 & 81 & 13 & 9 & 7 & 0 & 0 \\
\hline 12 & 1 & 1 & 1 & 3 & 6 & TS & 3 & 165 & 225 & 5 & 8 & 5 & 0 & 0 \\
\hline 12 & 1 & 1 & 1 & 4 & 5 & $-\mathrm{S}$ & 4 & 154 & 204 & 7 & 10 & 6 & 0 & 0 \\
\hline 12 & 1 & 1 & 2 & 2 & 6 & TS & 2 & 128 & 171 & 5 & 8 & 5 & 1 & 1 \\
\hline 12 & 1 & 1 & 2 & 3 & 5 & $\mathrm{TS}$ & 3 & 105 & 137 & 7 & 8 & 6 & 0 & 0 \\
\hline 12 & 1 & 1 & 2 & 4 & 4 & TS & 5 & 101 & 130 & 5 & 8 & 5 & 1 & 1 \\
\hline 12 & 1 & 1 & 3 & 3 & 4 & TS & 5 & 89 & 115 & 5 & 7 & 5 & 0 & 0 \\
\hline 12 & 1 & 2 & 2 & 2 & 5 & TS & 4 & 94 & 108 & 8 & 9 & 6 & 0 & 0 \\
\hline 12 & 1 & 2 & 2 & 3 & 4 & TS & 2 & 74 & 89 & 5 & 7 & 5 & 0 & 0 \\
\hline 12 & 1 & 2 & 3 & 3 & 3 & TS & 3 & 69 & 81 & 5 & 8 & 5 & 1 & 1 \\
\hline 12 & 2 & 2 & 2 & 3 & 3 & TS & 6 & 60 & 63 & 5 & 8 & 5 & 1 & 1 \\
\hline$\cdots$ & $\cdots$ & $\cdots$ & $\cdots$ & $\cdots$ & $\cdots$ & $\cdots$ & $\cdots$ & $\cdots$ & $\cdots$ & $\cdots$ & $\cdots$ & $\cdots$ & $\cdots$ & $\cdots$ \\
\hline 3192 & 37 & 39 & 456 & 1064 & 1596 & -- & 491 & 11 & 26 & 5 & 680 & 5 & 2 & 1 \\
\hline 3234 & 36 & 41 & 462 & 1078 & 1617 & $\mathrm{~T}-$ & 462 & 12 & 27 & 6 & 639 & 6 & 2 & 1 \\
\hline 3234 & 37 & 40 & 462 & 1078 & 1617 & -- & 491 & 11 & 26 & 5 & 680 & 5 & 2 & 1 \\
\hline 3234 & 38 & 39 & 462 & 1078 & 1617 & -- & 491 & 11 & 26 & 5 & 680 & 5 & 2 & 1 \\
\hline 3276 & 37 & 41 & 468 & 1092 & 1638 & -- & 491 & 11 & 26 & 5 & 680 & 5 & 2 & 1 \\
\hline 3318 & 37 & 42 & 474 & 1106 & 1659 & -- & 491 & 11 & 26 & 5 & 680 & 5 & 2 & 1 \\
\hline 3318 & 38 & 41 & 474 & 1106 & 1659 & -- & 491 & 11 & 26 & 5 & 680 & 5 & 2 & 1 \\
\hline 3318 & 39 & 40 & 474 & 1106 & 1659 & -- & 491 & 11 & 26 & 5 & 680 & 5 & 2 & 1 \\
\hline 3360 & 39 & 41 & 480 & 1120 & 1680 & -- & 491 & 11 & 26 & 5 & 680 & 5 & 2 & 1 \\
\hline 3402 & 40 & 41 & 486 & 1134 & 1701 & -- & 491 & 11 & 26 & 5 & 680 & 5 & 2 & 1 \\
\hline 3486 & 41 & 42 & 498 & 1162 & 1743 & $\mathrm{~T}-$ & 491 & 11 & 26 & 5 & 680 & 5 & 2 & 1 \\
\hline
\end{tabular}

Table 5: Results from the complete list of reflexive weights (available via WWW) 


\begin{tabular}{|c|c|c|c|c|c|c|c|c|c|c|c|c|c|c|}
\hline$d$ & $w_{1}$ & $w_{2}$ & $w_{3}$ & $w_{4}$ & $w_{5}$ & $\mathrm{TS}$ & $h_{11}$ & $h_{12}$ & $\# P$ & $\# V$ & $\# \bar{P}$ & $\# \bar{V}$ & $\Pi$ & $\bar{F}$ \\
\hline 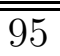 & $\bar{~} 15$ & $\bar{~} 17$ & $\bar{~} 18$ & $\bar{~} 19$ & 26 & -- & 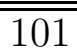 & $\overline{\overline{1}}$ & 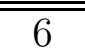 & 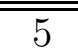 & $\bar{~} 126$ & $\overline{\overline{5}}$ & $\bar{~} \bar{l}$ & 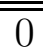 \\
\hline 47 & 3 & 4 & 5 & 14 & 21 & $-\mathrm{S}$ & 26 & 39 & 54 & 18 & 35 & 15 & $?$ & 0 \\
\hline 69 & 7 & 8 & 10 & 19 & 25 & $-\mathrm{S}$ & 59 & 10 & 16 & 13 & 75 & 21 & $?$ & $\overline{0}$ \\
\hline 97 & 7 & 8 & 11 & 26 & 45 & $-\mathrm{S}$ & 63 & 15 & 24 & 15 & 71 & 21 & $?$ & 1 \\
\hline 84 & 1 & 1 & 12 & 28 & 42 & $\mathrm{TS}$ & 11 & 491 & 680 & 5 & 26 & 5 & 1 & 1 \\
\hline 24 & 3 & 4 & 5 & 6 & 6 & $\mathrm{TS}$ & 10 & 34 & 36 & 8 & 12 & 7 & $?$ & 0 \\
\hline 26 & 3 & 4 & 5 & 7 & 7 & $-\mathrm{S}$ & 22 & 22 & 31 & 13 & 21 & 10 & $?$ & 0 \\
\hline 33 & 3 & 6 & 6 & 7 & 11 & -- & 19 & 37 & 34 & 7 & 22 & 6 & $?$ & 0 \\
\hline 36 & 3 & 6 & 6 & 10 & 11 & $\mathrm{~T}-$ & 19 & 49 & 38 & 7 & 22 & 6 & $?$ & 0 \\
\hline 26 & 3 & 4 & 5 & 6 & 8 & $-\mathrm{S}$ & 14 & 24 & 32 & 14 & 19 & 10 & $?$ & 1 \\
\hline 36 & 5 & 7 & 7 & 8 & 9 & -- & 30 & 12 & 19 & 10 & 28 & 9 & $?$ & 1 \\
\hline 39 & 3 & 6 & 9 & 10 & 11 & $\mathrm{TS}$ & 17 & 41 & 33 & 12 & 22 & 13 & $?$ & 1 \\
\hline 52 & 4 & 6 & 8 & 11 & 23 & $\mathrm{~T}-$ & 29 & 33 & 34 & 9 & 36 & 8 & $?$ & 1 \\
\hline 34 & 3 & 6 & 7 & 8 & 10 & $-\mathrm{S}$ & 18 & 20 & 27 & 13 & 23 & 12 & $?$ & 2 \\
\hline 44 & 4 & 8 & 9 & 10 & 13 & -- & 29 & 17 & 22 & 9 & 31 & 9 & $?$ & 2 \\
\hline 55 & 3 & 10 & 13 & 14 & 15 & $\mathrm{TS}$ & 28 & 16 & 23 & 12 & 35 & 14 & $?$ & 2 \\
\hline 63 & 7 & 9 & 14 & 15 & 18 & $\mathrm{~T}-$ & 44 & 8 & 15 & 6 & 37 & 6 & $?$ & 2 \\
\hline 5 & 1 & 1 & 1 & 1 & 1 & $\mathrm{TS}$ & 1 & 101 & 126 & 5 & 6 & 5 & 0 & 0 \\
\hline 10 & 1 & 1 & 1 & 3 & 4 & $-\mathrm{S}$ & 4 & 126 & 165 & 10 & 9 & 7 & 0 & 0 \\
\hline 25 & 1 & 5 & 5 & 6 & 8 & $\mathrm{~T}-$ & 17 & 49 & 65 & 7 & 15 & 7 & 0 & 0 \\
\hline 26 & 1 & 5 & 5 & 7 & 8 & -- & 19 & 49 & 65 & 9 & 19 & 7 & 0 & 0 \\
\hline 20 & 2 & 3 & 4 & 4 & 7 & $-\mathrm{S}$ & 13 & 45 & 51 & 10 & 14 & 8 & 1 & 0 \\
\hline 20 & 2 & 3 & 5 & 5 & 5 & $\mathrm{TS}$ & 6 & 48 & 50 & 8 & 11 & 6 & 1 & 0 \\
\hline 30 & 2 & 5 & 6 & 6 & 11 & -- & 27 & 39 & 45 & 7 & 25 & 6 & 1 & 0 \\
\hline 36 & 2 & 5 & 6 & 6 & 17 & $\mathrm{~T}-$ & 24 & 54 & 60 & 7 & 25 & 6 & 1 & 0 \\
\hline 8 & 1 & 1 & 2 & 2 & 2 & $\mathrm{TS}$ & 2 & 86 & 105 & 5 & 7 & 5 & 1 & 1 \\
\hline 13 & 1 & 1 & 2 & 4 & 5 & $-\mathrm{S}$ & 6 & 108 & 141 & 12 & 11 & 8 & 1 & 1 \\
\hline 35 & 2 & 7 & 8 & 9 & 9 & -- & 35 & 23 & 33 & 11 & 30 & 8 & 1 & 1 \\
\hline 40 & 4 & 5 & 9 & 10 & 12 & $\mathrm{~T}-$ & 22 & 18 & 25 & 7 & 20 & 7 & 1 & 1 \\
\hline 19 & 2 & 3 & 4 & 5 & 5 & $-\mathrm{S}$ & 11 & 33 & 43 & 14 & 14 & 9 & 2 & 1 \\
\hline 27 & 2 & 3 & 4 & 9 & 9 & $\mathrm{TS}$ & 14 & 44 & 56 & 9 & 13 & 7 & 2 & 1 \\
\hline 36 & 4 & 4 & 6 & 9 & 13 & -- & 31 & 31 & 33 & 6 & 29 & 6 & 2 & 1 \\
\hline 40 & 4 & 4 & 6 & 9 & 17 & $\mathrm{~T}-$ & 26 & 38 & 39 & 7 & 29 & 6 & 2 & 1 \\
\hline 14 & 2 & 2 & 3 & 3 & 4 & $\mathrm{TS}$ & 5 & 51 & 57 & 10 & 10 & 7 & 2 & 2 \\
\hline 19 & 2 & 3 & 3 & 4 & 7 & $-\mathrm{S}$ & 11 & 39 & 51 & 14 & 16 & 9 & 2 & 2 \\
\hline 30 & 3 & 5 & 5 & 6 & 11 & -- & 33 & 21 & 33 & 6 & 25 & 6 & 2 & 2 \\
\hline 35 & 3 & 5 & 5 & 6 & 16 & $\mathrm{~T}-$ & 26 & 28 & 42 & 7 & 25 & 6 & 2 & 2 \\
\hline 28 & 4 & 5 & 5 & 6 & 8 & $-\mathrm{S}$ & 18 & 20 & 27 & 10 & 18 & 8 & 3 & 2 \\
\hline 36 & 4 & 6 & 8 & 9 & 9 & $\mathrm{TS}$ & 23 & 23 & 26 & 6 & 16 & 6 & 3 & 2 \\
\hline 40 & 4 & 7 & 7 & 10 & 12 & -- & 28 & 16 & 23 & 7 & 25 & 6 & 3 & 2 \\
\hline 42 & 6 & 7 & 7 & 10 & 12 & $\mathrm{~T}-$ & 35 & 11 & 19 & 6 & 23 & 6 & 3 & 2 \\
\hline
\end{tabular}

Table 6: Examples from the long list of weights with different features 


\begin{tabular}{|c|c|c|c|c|c|c|c|c|c|c|c|}
\hline \multirow{2}{*}{$\frac{d}{4}$} & \multicolumn{4}{|c|}{$\begin{array}{lllll}w_{1} & w_{2} & w_{3} & w_{4} \\
\end{array}$} & \multirow{2}{*}{$\begin{array}{l}\mathrm{S} \\
\mathrm{S}\end{array}$} & \multicolumn{2}{|c|}{$\# P \# V$} & \multicolumn{2}{|c|}{$\# \bar{P} \# \bar{V}$} & \multirow{2}{*}{$\frac{\Pi}{0}$} & \multirow{2}{*}{ F } \\
\hline & 1 & 1 & 1 & & & 35 & 4 & 5 & 4 & & \\
\hline 5 & 1 & 1 & 1 & 2 & $\mathrm{~S}$ & 34 & 6 & 6 & 5 & 0 & 0 \\
\hline 6 & 1 & 1 & 1 & 3 & S & 39 & 4 & 6 & 4 & 0 & 0 \\
\hline 6 & 1 & 1 & 2 & 2 & S & 30 & 4 & 6 & 4 & 1 & 1 \\
\hline 7 & 1 & 1 & 2 & 3 & S & 31 & 7 & 8 & 6 & 1 & 1 \\
\hline 8 & 1 & 2 & 2 & 3 & $\mathrm{~S}$ & 24 & 6 & 8 & 5 & 0 & 0 \\
\hline 8 & 1 & 1 & 2 & 4 & S & 35 & 4 & 7 & 4 & 1 & 1 \\
\hline 9 & 1 & 2 & 3 & 3 & S & 23 & 6 & 8 & 5 & 1 & 1 \\
\hline 9 & 1 & 1 & 3 & 4 & S & 33 & 5 & 9 & 5 & 1 & 1 \\
\hline 10 & 1 & 2 & 2 & 5 & $\mathrm{~S}$ & 28 & 4 & 8 & 4 & 0 & 0 \\
\hline 10 & 1 & 2 & 3 & 4 & S & 23 & 7 & 11 & 6 & 1 & 1 \\
\hline 10 & 1 & 1 & 3 & 5 & S & 36 & 5 & 9 & 5 & 1 & 1 \\
\hline 11 & 1 & 2 & 3 & 5 & $\mathrm{~S}$ & 24 & 8 & 13 & 7 & 1 & 1 \\
\hline 12 & 1 & 2 & 3 & 6 & S & 27 & 4 & 9 & 4 & 1 & 1 \\
\hline 12 & 1 & 2 & 4 & 5 & S & 24 & 5 & 12 & 5 & 1 & 1 \\
\hline 12 & 1 & 3 & 4 & 4 & S & 21 & 4 & 9 & 4 & 1 & 1 \\
\hline 12 & 2 & 3 & 3 & 4 & S & 15 & 4 & 9 & 4 & 2 & 2 \\
\hline 12 & 1 & 1 & 4 & 6 & $\mathrm{~S}$ & 39 & 4 & 9 & 4 & 1 & 1 \\
\hline 12 & 2 & 2 & 3 & 5 & S & 17 & 5 & 11 & 5 & 1 & 1 \\
\hline 13 & 1 & 3 & 4 & 5 & S & 20 & 7 & 15 & 7 & 1 & 1 \\
\hline 14 & 1 & 2 & 4 & 7 & $\mathrm{~S}$ & 27 & 5 & 12 & 5 & 1 & 1 \\
\hline 14 & 2 & 3 & 4 & 5 & S & 13 & 7 & 16 & 7 & 3 & 2 \\
\hline 14 & 2 & 2 & 3 & 7 & S & 19 & 5 & 11 & 5 & 1 & 1 \\
\hline 15 & 1 & 2 & 5 & 7 & $\mathrm{~S}$ & 26 & 6 & 17 & 6 & 1 & 1 \\
\hline 15 & 1 & 3 & 4 & 7 & S & 22 & 6 & 17 & 6 & 1 & 1 \\
\hline 15 & 1 & 3 & 5 & 6 & $\mathrm{~S}$ & 21 & 5 & 15 & 5 & 1 & 1 \\
\hline 15 & 2 & 3 & 5 & 5 & S & 14 & 6 & 11 & 5 & 1 & 0 \\
\hline 15 & 3 & 3 & 4 & 5 & $\mathrm{~S}$ & 12 & 5 & 12 & 5 & 1 & 1 \\
\hline 16 & 1 & 2 & 5 & 8 & $\mathrm{~S}$ & 28 & 5 & 14 & 5 & 1 & 1 \\
\hline 16 & 1 & 3 & 4 & 8 & S & 24 & 5 & 12 & 5 & 1 & 1 \\
\hline 16 & 1 & 4 & 5 & 6 & S & 19 & 6 & 17 & 6 & 1 & 1 \\
\hline 16 & 2 & 3 & 4 & 7 & S & 14 & 6 & 18 & 6 & 2 & 1 \\
\hline 17 & 2 & 3 & 5 & 7 & $\mathrm{~S}$ & 13 & 8 & 20 & 8 & 2 & 1 \\
\hline 18 & 1 & 2 & 6 & 9 & $\mathrm{~S}$ & 30 & 4 & 12 & 4 & 1 & 1 \\
\hline 18 & 1 & 3 & 5 & 9 & $\mathrm{~S}$ & 24 & 5 & 15 & 5 & 1 & 1 \\
\hline 18 & 1 & 4 & 6 & 7 & $\mathrm{~S}$ & 19 & 6 & 20 & 6 & 1 & 1 \\
\hline 18 & 2 & 3 & 4 & 9 & $\mathrm{~S}$ & 16 & 5 & 14 & 5 & 2 & 1 \\
\hline 18 & 2 & 3 & 5 & 8 & $\mathrm{~S}$ & 14 & 6 & 20 & 6 & 2 & 1 \\
\hline 18 & 3 & 4 & 5 & 6 & $\mathrm{~S}$ & 10 & 6 & 17 & 6 & $?$ & 1 \\
\hline 19 & 3 & 4 & 5 & 7 & $\mathrm{~S}$ & 9 & 7 & 24 & 8 & $?$ & 1 \\
\hline 20 & 1 & 4 & 5 & 10 & $\mathrm{~S}$ & 23 & 4 & 13 & 4 & 1 & 1 \\
\hline 20 & 2 & 3 & 5 & 10 & $\mathrm{~S}$ & 16 & 5 & 14 & 5 & 2 & 1 \\
\hline 20 & 2 & 5 & 6 & 7 & - & 11 & 5 & 23 & 5 & 3 & 2 \\
\hline 20 & 2 & 4 & 5 & 9 & - & 13 & 4 & 23 & 4 & 1 & 1 \\
\hline 20 & 3 & 4 & 5 & 8 & $\mathrm{~S}$ & 10 & 6 & 22 & 6 & $?$ & 0 \\
\hline 21 & 1 & 3 & 7 & 10 & - & 24 & 4 & 24 & 4 & 1 & 1 \\
\hline 21 & 1 & 5 & 7 & 8 & - & 18 & 5 & 24 & 5 & 1 & 1 \\
\hline 21 & 2 & 3 & 7 & 9 & $\mathrm{~S}$ & 14 & 6 & 23 & 6 & 2 & 1 \\
\hline
\end{tabular}

\begin{tabular}{|c|c|c|c|c|c|c|c|c|c|c|c|}
\hline$d$ & $w_{1}$ & $w_{2}$ & $w_{3}$ & $w_{4}$ & $S$ & $\# P$ & $\# V$ & $\# \bar{P}$ & $\# \bar{V}$ & $\Pi$ & $\mathrm{F}$ \\
\hline 21 & 3 & 5 & 6 & & - & 9 & 5 & 21 & 5 & ? & 1 \\
\hline 22 & 1 & 3 & 7 & 11 & - & 25 & 5 & 20 & 5 & 1 & 1 \\
\hline 22 & 1 & 4 & 6 & 11 & S & 22 & 6 & 20 & 6 & 1 & 1 \\
\hline 22 & 2 & 4 & 5 & 11 & - & 14 & 5 & 19 & 5 & 1 & 1 \\
\hline 24 & 1 & 3 & 8 & 12 & S & 27 & 4 & 15 & 4 & 1 & 1 \\
\hline 24 & 1 & 6 & 8 & 9 & $\mathrm{~S}$ & 18 & 5 & 24 & 5 & 1 & 1 \\
\hline 24 & 2 & 3 & 8 & 11 & - & 15 & 4 & 27 & 4 & 1 & 0 \\
\hline 24 & 2 & 3 & 7 & 12 & S & 16 & 5 & 20 & 5 & 2 & 1 \\
\hline 24 & 3 & 4 & 5 & 12 & S & 12 & 5 & 18 & 5 & ? & 0 \\
\hline 24 & 3 & 4 & 7 & 10 & $\mathrm{~S}$ & 10 & 5 & 26 & 6 & 2 & 1 \\
\hline 24 & 3 & 6 & 7 & 8 & - & 9 & 4 & 21 & 4 & $?$ & 1 \\
\hline 24 & 4 & 5 & 6 & 9 & $\mathrm{~S}$ & 8 & 5 & 26 & 6 & $?$ & 1 \\
\hline 25 & 4 & 5 & 7 & 9 & - & 7 & 5 & 32 & 6 & $?$ & 1 \\
\hline 26 & 1 & 5 & 7 & 13 & - & 21 & 5 & 24 & 5 & 1 & 1 \\
\hline 26 & 2 & 3 & 8 & 13 & - & 16 & 5 & 23 & 5 & 1 & 0 \\
\hline 26 & 2 & 5 & 6 & 13 & - & 13 & 5 & 23 & 5 & 2 & 1 \\
\hline 27 & 2 & 5 & 9 & 11 & - & 11 & 6 & 32 & 6 & 2 & 1 \\
\hline 27 & 5 & 6 & 7 & 9 & - & 6 & 5 & 30 & 6 & ? & 0 \\
\hline 28 & 1 & 4 & 9 & 14 & - & 24 & 4 & 24 & 4 & 1 & 1 \\
\hline 28 & 3 & 4 & 7 & 14 & S & 12 & 5 & 18 & 5 & 2 & 1 \\
\hline 28 & 4 & 6 & 7 & 11 & - & 7 & 4 & 35 & 4 & ? & 1 \\
\hline 30 & 1 & 4 & 10 & 15 & $\mathrm{~S}$ & 25 & 5 & 20 & 5 & 1 & 1 \\
\hline 30 & 1 & 6 & 8 & 15 & $\mathrm{~S}$ & 21 & 5 & 24 & 5 & 1 & 1 \\
\hline 30 & 2 & 3 & 10 & 15 & S & 18 & 4 & 18 & 4 & 1 & 0 \\
\hline 30 & 2 & 6 & 7 & 15 & - & 13 & 4 & 23 & 4 & 1 & 1 \\
\hline 30 & 3 & 4 & 10 & 13 & - & 10 & 5 & 35 & 5 & 2 & 1 \\
\hline 30 & 4 & 5 & 6 & 15 & S & 10 & 5 & 20 & 5 & $?$ & 0 \\
\hline 30 & 5 & 6 & 8 & 11 & - & 6 & 4 & 39 & 4 & $?$ & 1 \\
\hline 32 & 2 & 5 & 9 & 16 & - & 13 & 5 & 29 & 5 & 2 & 1 \\
\hline 32 & 4 & 5 & 7 & 16 & - & 9 & 5 & 27 & 5 & $?$ & 0 \\
\hline 33 & 3 & 5 & 11 & 14 & - & 9 & 4 & 39 & 4 & 2 & 1 \\
\hline 34 & 3 & 4 & 10 & 17 & - & 11 & 6 & 31 & 6 & 2 & 1 \\
\hline 34 & 4 & 6 & 7 & 17 & - & 8 & 5 & 31 & 5 & $?$ & 1 \\
\hline 36 & 1 & 5 & 12 & 18 & - & 24 & 4 & 24 & 4 & 1 & 1 \\
\hline 36 & 3 & 4 & 11 & 18 & - & 12 & 4 & 30 & 4 & 2 & 1 \\
\hline 36 & 7 & 8 & 9 & 12 & - & 5 & 4 & 35 & 4 & $?$ & 0 \\
\hline 38 & 3 & 5 & 11 & 19 & - & 10 & 5 & 35 & 5 & 2 & 1 \\
\hline 38 & 5 & 6 & 8 & 19 & - & 7 & 5 & 35 & 5 & $?$ & 1 \\
\hline 40 & 5 & 7 & 8 & 20 & - & 8 & 4 & 28 & 4 & $?$ & 0 \\
\hline 42 & 1 & 6 & 14 & 21 & S & 24 & 4 & 24 & 4 & 1 & 1 \\
\hline 42 & 2 & 5 & 14 & & - & 15 & 4 & 27 & 4 & 1 & 0 \\
\hline 42 & 3 & 4 & 14 & 21 & $\mathrm{~S}$ & 13 & 5 & 26 & 5 & 2 & 1 \\
\hline 44 & 4 & 5 & 13 & & - & 9 & 4 & 39 & 4 & 2 & 1 \\
\hline 48 & 3 & 5 & 16 & & - & 12 & 4 & 30 & 4 & 2 & 1 \\
\hline 50 & 7 & 8 & 10 & 25 & - & 6 & 4 & 39 & 4 & $?$ & 1 \\
\hline 54 & 4 & 5 & 18 & 27 & - & 10 & 5 & 35 & 5 & 2 & 1 \\
\hline 66 & 5 & 6 & 22 & & - & 9 & 4 & 39 & 4 & 2 & 1 \\
\hline
\end{tabular}

Table 7: Elliptic fibration data for the $95 \mathrm{~K} 3$ weights (58 are spanning; all are transversal) 


\begin{tabular}{||c||c|c|c||c||}
\hline \hline & $\# F=0$ & $\# F=1$ & $\# F=2$ & total \\
\hline \hline$\# \Pi=?$ & 7 & 11 & 0 & 18 \\
\hline$\# \Pi=0$ & 5 & & & 5 \\
\hline$\# \Pi=1$ & 5 & 42 & & 47 \\
\hline$\# \Pi=2$ & 0 & 22 & 1 & 23 \\
\hline$\# \Pi=3$ & 0 & 0 & 2 & 2 \\
\hline \hline total & 17 & 75 & 3 & 95 \\
\hline \hline
\end{tabular}

Table 8: Numbers of reflexive projections $(\# \Pi)$ and facets $(\# F)$ for the K3 weights

\begin{tabular}{||c||c|c|c||c||}
\hline \hline Spectra & MNPs & no mirror & together & $\chi=0$ \\
\hline \hline$W P^{4}$ & 2,780 & 669 & 3,449 & 63 \\
\hline orbifolds & 3,537 & 781 & 4,318 & 72 \\
\hline MNPs & 10,237 & 3,884 & 14,121 & 103 \\
\hline \hline total & 10,410 & 3,806 & 14,216 & 104 \\
\hline \hline
\end{tabular}

Table 9: Hodge numbers for transversal weights, orbifolds and maximal Newton polyhedra

Acknowledgements. A.C.A. is supported by the Robert A. Welch Foundation and by the NSF under grant PHY/9511632. M.K. is supported by the Austrian Research Fund under grant number P10641-PHY. M.M. is supported by the United States National Science Foundation under grants PHY-9404057 and PHY-9457916. H.S. is supported by the Austrian National Bank under grant number 5674. It is a pleasure to acknowledge useful conversations with Per Berglund, Philip Candelas and Anamaria Font. Most of this work was done while M.M. was at the University of Texas at Austin. 


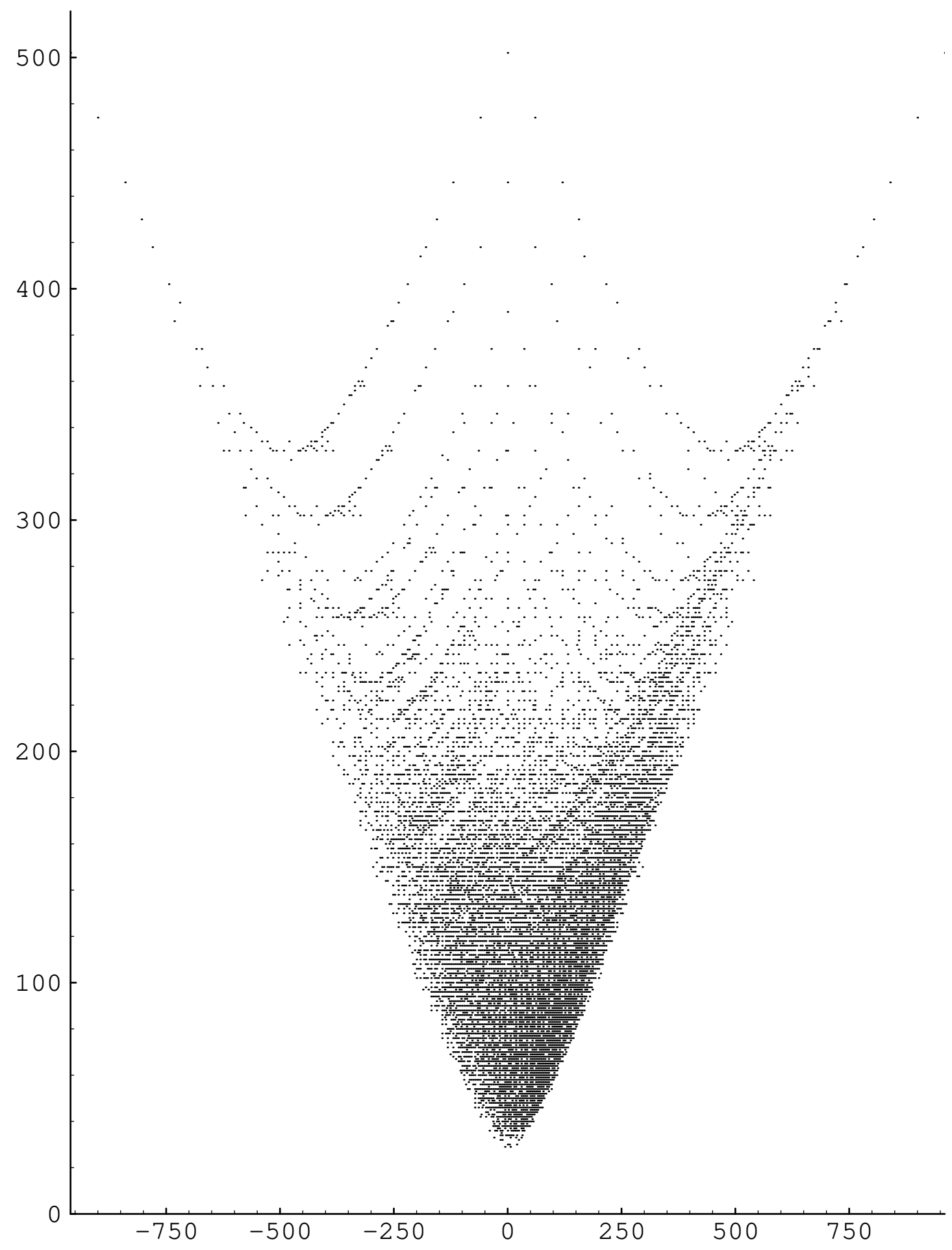

Figure 4: $b_{11}+b_{21}$ vs $\chi_{E}$ for single weight systems 


\section{References}

[1] P. Candelas, G. Horowitz, A. Strominger and E. Witten, Vacuum configurations for superstrings,Nucl. Phys. B258 (1985) 46.

[2] P. Candelas, A. Dale, C. Lütken, and R. Schimmrigk, Complete intersection Calabi-Yau manifolds,Nucl. Phys. B298 (1988) 493.

[3] S. Yau and G. Tian, in Proc. Argonne Symposium on Anomalies, Geometry and Topology, ed. W. Bardeen, A. White, World Scientific, Singapore, 1985.

[4] M.Kreuzer, H.Skarke, No mirror symmetry in Landau-Ginzburg spectra!, hep-th/9205004 Nucl. Phys. B388 (1992) 113.

[5] A.Klemm, R.Schimmrigk, Landau-Ginzburg String Vacua, hep-th/9204060, Nucl. Phys. B411 (1994) 559.

[6] V.V.Batyrev, Dual polyhedra and mirror symmetry for Calabi-Yau hypersurfaces in toric varieties, alg-geom/9310003, J. Alg. Geom. 3 (1994) 493.

[7] M.Kreuzer, H.Skarke, On the classification of reflexive polyhedra, hep-th/9512204, TUW-95-26.

[8] H.Skarke, Weight systems for toric Calabi-Yau varieties and reflexivity of Newton polyhedra, Mod. Phys. Lett. A11 (1996) 1637.

[9] S. Kachru and C. Vafa, Exact results for $N=2$ compactifications of heterotic strings, Nucl. Phys. B450 (1995) 69.

[10] N. Seiberg and E. Witten, Electric-magnetic duality, monopole condensation, and confinement in $N=2$ supersymmetric Yang-Mills theory,Nucl. Phys. B426 (1994) 19.

N. Seiberg and E. Witten, Monopoles, duality, and chiral symmetry breaking in $N=2$ supersymmetric QCD,Nucl. Phys. B431 (1994) 484.

[11] A. Klemm, W. Lerche and P. Mayr, K3 fibrations and heterotic-Type II String Duality, Phys. Lett. B357 (1995) 313.

[12] S.Hosono, B.H.Lian, S.-T. Yau, Calabi-Yau Varieties and Pencils of K3 Surfaces, alg-geom/9603020.

[13] P. Aspinwall and J. Louis, On the ubiquity of $K 3$ fibrations in string duality, Phys. Lett. B369 (1996) 233.

[14] P. Candelas and A. Font, Duality between the webs of heterotic and Type II vacua, hep-th/9603170, UTTG-04-96.

[15] W.Fulton, Introduction to Toric Varieties, Princeton University Press (1993).

[16] D.Cox, The homogeneous coordinate ring or a toric variety, J. Alg. Geom. 4 (1995) 17.

[17] M.Audin, The Topology of Torus Actions on Symplectic Manifolds, Progress in Math. 93, Birkhäuser, 1991.

[18] E.Witten, Phases of $N=2$ theories in two dimensions, Nucl. Phys. B403 (1993) 159.

[19] M. Kreuzer and H. Skarke, All Abelian Symmetries of Landau-Ginzburg Potentials, Nucl. Phys. B405 (1993) 305. 